\title{
Numerical Analysis of Orbital Angular Momentum Based Next Generation Optical SDM Communications System
}

\author{
Besma Hammami, Habib Fathallah, and Houria Rezig
}

\begin{abstract}
In this paper, we make a theoretical and numerical analysis of a transmission chain based on an Orbital Angular Momentum (OAM) technique in the case of ideal channel. In order to generate an OAM beam, we define a theoretical OAM encoder (vortex generator) similar to the Spiral Phase Pattern (SPP) in practice, converts a flat Gaussian phase front into a helix. We then multiplex several OAM channels, all modulated with QPSK streams of data and then decode specific modes and measure the overall performance in terms of signal to noise ratio (SNR) and bit error rate (BER).
\end{abstract}

Index Terms-Orbital angular momentum (OAM), OAM beam, vortex generator, theoretical OAM encoder.

\section{INTRODUCTION}

There are two particularly important ways in which a light beam can rotate: if every polarization vector rotates, the light has spin; if the phase structure rotates, the light has orbital angular momentum (OAM). Recently, much attention has been focused and taken more interest in the orbital angular momentum (OAM). The OAM of a light beam known as an optical vortex can exist in one of an infinite number of states and may be used to carry information. The information carrying OAM beams can achieve an increase in the capacity of optical communication system and spectral efficiency [1]-[4].

Using OAM beams for multiplexing can be regarded as analogous to various other multiplexing technologies, such as wavelength-division multiplexing (WDM) [5], [6], optical time-division multiplexing (OTDM) [7], spatial-division multiplexing (SDM) using multicore fibers [8] and mode-division multiplexing (MDM) [9].

Orbital angular momentum has given rise to many developments in optical manipulation, optical trapping, imaging, astronomy and quantum information processing. In addition to these established areas, OAM has recently seen application in free-space information transfer and communications [10], [11]. Although results have been achieved in free- space, there is significant interest in the potential to use OAM for MDM in fiber [12]-[14].

In the work reported here, we focus our attention on three blocks of an optical communication system that is based on orbital angular momentum, which are; the OAM encoder, the multiplexing, and de-multiplexing. We present a theoretical

Manuscript received February 14, 2015; revised April 27, 2015.

Besma Hammami and Houria Rezig are with the Laboratoire Syscom, National School of Engineer Tunis, University de Manar, Tunis, Tunisia (e-mail: hammamibesma6@gmail.com,hourezg@yahoo.fr).

Habib Fathallah is with the KACST-TIC in Radio Frequency and Photonics for the e-Society, and the Electrical Engineering Department, King Saud University, Riyadh, Saudi Arabia (e-mail: hfathallah@ksu.edu.sa). and numerical simulation analysis (using Matlab) in which we can simulate our optical chain and evaluate the bit error rate (BER) performance of the system.

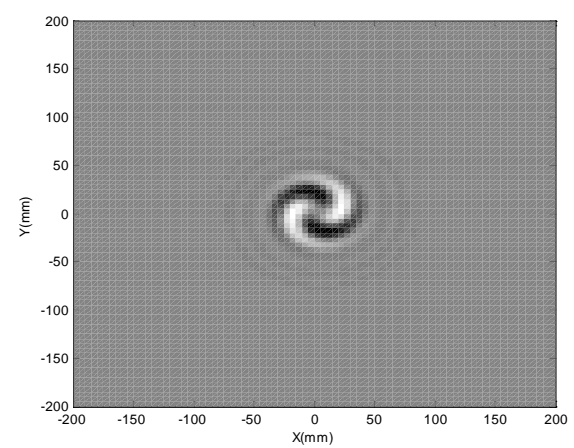

(a)

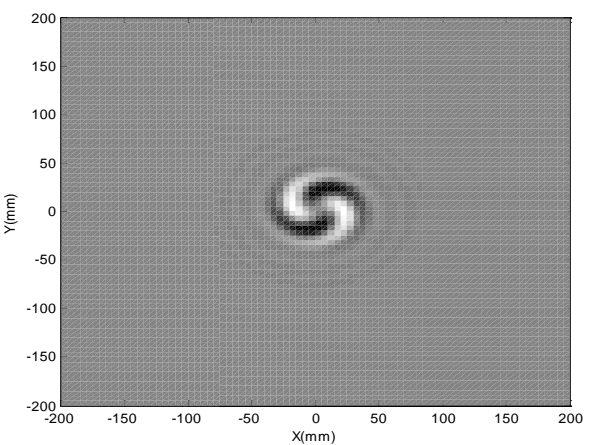

(b)

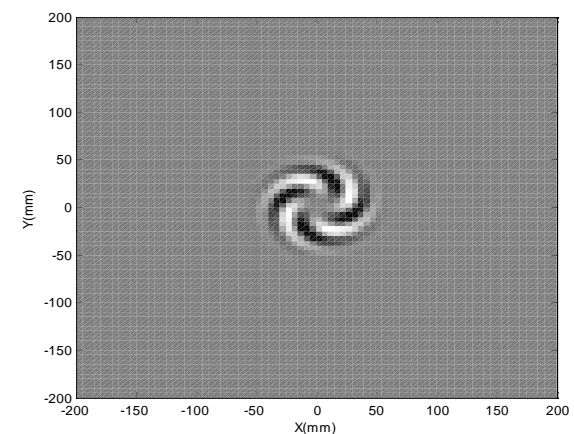

(c)

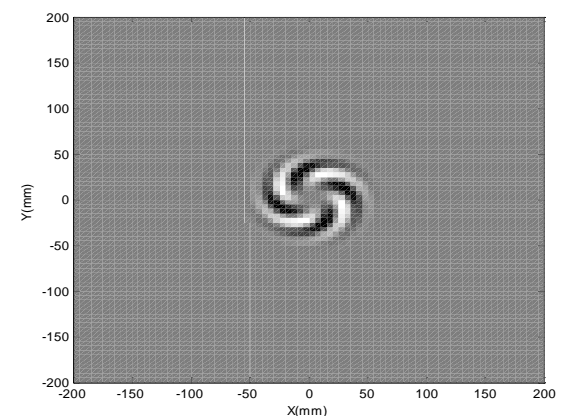

Fig. 1. The electric field intensity distribution of a Gaussian vortex beam (in space, mm unit in $X$ and $Y$ axis) for different values of the topological charge «l; (a) $\mathrm{l}=2$; (b) $\mathrm{l}=-2$; (c) $\mathrm{l}=4$; (d) $\mathrm{l}=-4$. 

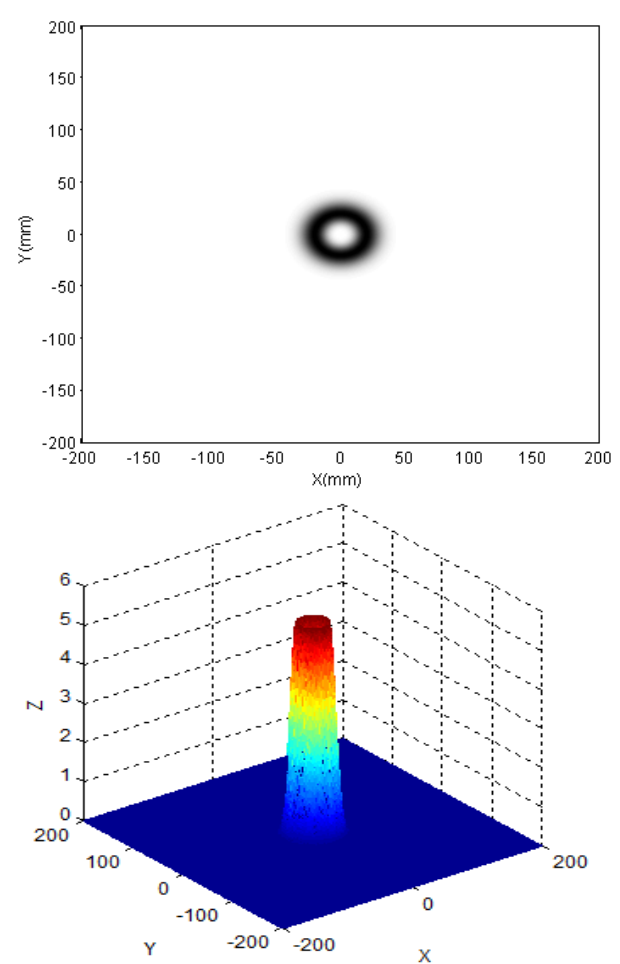

(a)

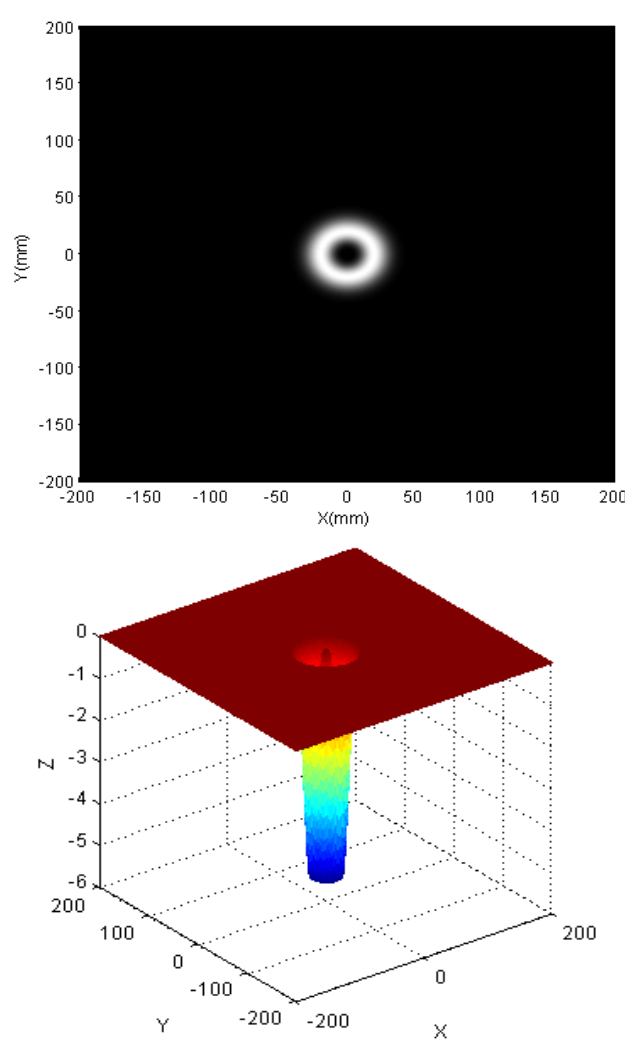

(b)

Fig. 2. The orbital angular momentum density distribution of Gaussian vortex beams (a) $l=4$; (b) $l=-4$.

This paper is organized as follows: In the second section, we present a mathematical model of the optical field and the orbital angular momentum density distribution of Gaussian vortex beams. In the third section we introduce the generation of an OAM beam using an OAM encoder and the multiplexing/de-multiplexing setup. In the fourth section we develop a theoretical and numerical simulation of an optical communication setup that exploits OAM beams for a transmission over a channel assumed to be ideal, and then we evaluate the performance of the proposed system through the bit error rate (BER).

\section{The Orbital Angular Momentum Density DisTRIBUTION OF GAUSSIAN VORTEX BEAMS: MATHEMATICAL MODEL}

\section{A. The Optical Field Distribution of Gaussian Vortex Beams}

A Gaussian vortex beam is a kind of typical vortex beam which is also called a helix beam. The transverse vortex optical field of a vortex beam is the optical field with the phase of a beam transverse section moving around the vortex center helically [15]. The electric field intensity of a Gaussian vortex beam can be expressed as [16]:

$$
E(x, y, z, t)=E_{0} \frac{\omega_{0}}{\omega(z)}\left(\frac{\sqrt{x^{2}+y^{2}}}{\omega(z)}\right)^{|l|} \exp \left(-\frac{x^{2}+y^{2}}{\omega(z)^{2}}\right) \exp [i \varphi(x, y, z, t)]
$$

where the phase term $\varphi(x, y, z, t)$ can be expressed as follows:

$$
\begin{aligned}
\varphi(x, y, z, t)= & -(|l|+1) \arctan \frac{2 z}{k \omega_{0}^{2}}+\frac{k\left(x^{2}+y^{2}\right)}{2 R(z)}+ \\
& l \cdot \arctan \left(\frac{y}{x}\right)+k z-\omega t
\end{aligned}
$$

The spot radius of the beam at position $\mathrm{z}$ is:

$$
\omega(z)=\omega_{0} \sqrt{1+\left(\frac{z}{z_{0}}\right)^{2}}
$$

The radius of curvature of the wave front is:

$$
R(z)=z\left(\frac{z}{z_{0}}+\frac{z_{0}}{z}\right)
$$

where $\mathrm{Z}_{0}$ is the Rayleigh range: $Z_{0}=\frac{1}{2} k \omega_{0}^{2}$

Integrating equations (2), (3) and (4) in equation (1), with $\lambda=1550 \mathrm{~nm}$, we developed a MATLAB program to simulate the electric field intensity of a Gaussian vortex beam as a function of the topological charge "l" (Fig. 1).

We can see in Fig. 1 that the wave fronts of Gaussian vortex beams rotate around the vortex center, and optical strength is zero at the vortex center.

The vortex characteristic is different when index "l" is different. When the absolute value of index "l" increases (Fig. 1(a), Fig. 1(c)), the spiral distribution characteristics of optical strength become complex, and when indexes "l" are mutual opposite numbers of the two beams, their spiral directions of optical strength distributions are opposite (Fig. 1(b), Fig. 1(d)).

\section{B. The Orbital Angular Momentum Density Distribution of Gaussian Vortex Beams}

The orbital angular momentum density of a Gaussian vortex beam can be presented by the following equation [16]:

$$
M_{z}=\frac{l \omega \varepsilon_{0}}{\pi^{2}+z^{2} \lambda^{2}} * \exp \left[\frac{-2 \pi^{2}\left(x^{2}+y^{2}\right)}{\omega_{0}^{2}\left(\pi^{2}+z^{2} \lambda^{2}\right.}\right] * \pi^{(2|l|+2)} *\left[\frac{x^{2}+y^{2}}{\omega_{0}^{2}\left(\pi^{2}+z^{2} \lambda^{2}\right)}\right]^{|l|}(5)
$$

Using the same parameters of the equation (1), we simulated the orbital angular momentum density distribution of Gaussian vortex beams.

The distribution of the orbital angular momentum of a 
Gaussian vortex beam is in a ring domain, and distribution shape is concave. In the center of the ring domain, orbital angular momentum density is maximal, and gradually decreases to zero from the center to the side (Fig. 2(a)). In Fig 2 (b), when indexes (l) are mutual opposite numbers of the beam, the values of their orbital angular momentum are equal, but the directions are opposite.
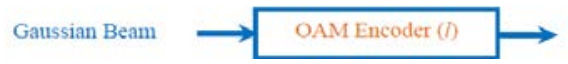

(a)
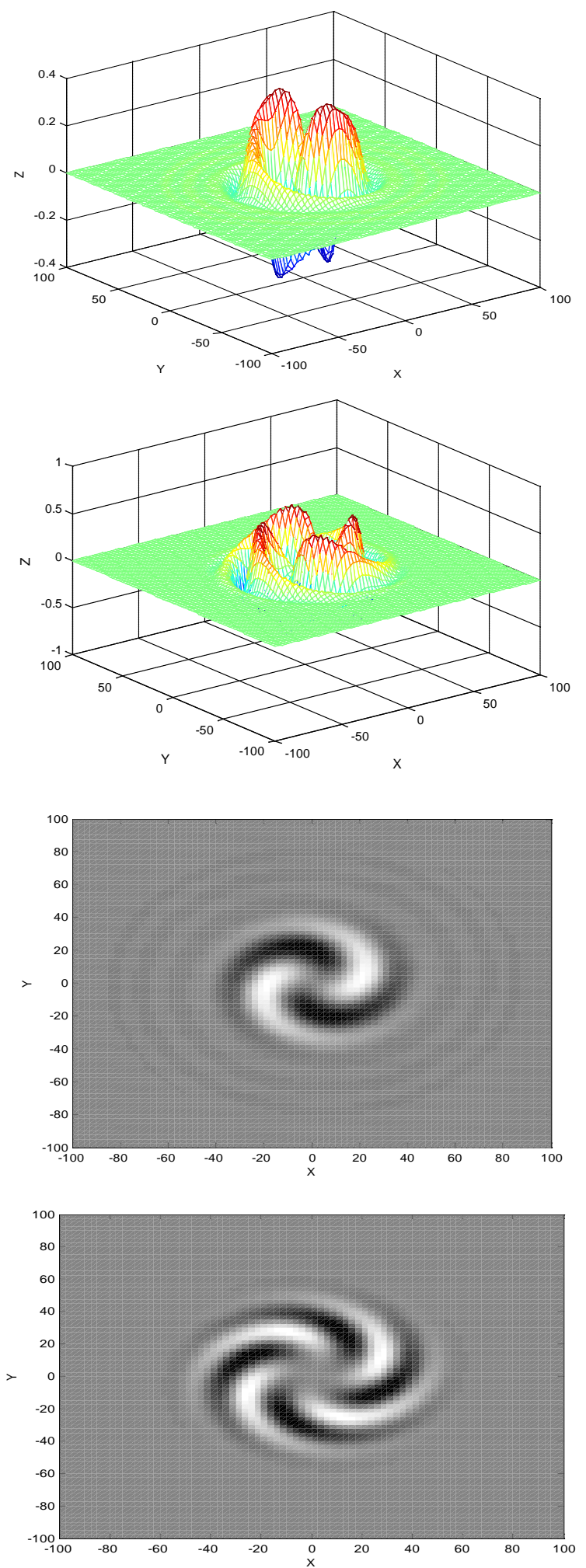

(b)

Fig. 3. (a) OAM Encoder; (b) Generation of OAM beams using an OAM Encoder with topological charge " $l=2$ " and " $l=4$ ".

\section{SYSTEM MODEL}

Unlike spin angular momentum, for which only two states are possible, the OAM state of a photon can take any integer value. This property may be exploited in the context of optical communications.

The orthogonality among beams with different OAM states allows to enable a variety of optical communication applications, such as multiplexing/de-multiplexing of information-carrying OAM beams to achieve an increase in capacity [11].

The OAM data transmission used here is based on quadrature-phase-shift-keying (QPSK) data at a single wavelength and carrying four OAM beams with different topological charge $l$.

The three key enablers for our demonstration are (i) a generation of OAM beams using an OAM encoder; (ii) a multiplexing setup; and (iii) a de-multiplexing setup.

\section{A. Generation of OAM Beams: OAM Encoder}

In this section, we will exploit the equations presented and simulated in the previous section, to develop a theoretical OAM encoder allows to generate OAM beams. The principle of this encoder based on the conversion of a flat phase front in a helix, as an adjustable spiral phase plate. An OAM encoder with number $l$ can be characterized by its code transfer function $\varphi_{1}(x, y, z)$.

An OAM beamU $(x, y, z)$ can be formed by integrating a Gaussian beam $G(x, y, z)$ into the OAM encoder (Fig. 3). The output of an arbitrary encoder $l$ denoted as $U_{l}(x, y, z)$ is the convolution between the signal $G(x, y, z)$ andtheencodertransfer function, achieved in " $z$ " and given by the following equation:

$$
U_{l}(x, y, z, t)=G(x, y, z) * \exp \left(i \varphi_{l}(x, y, z)\right)
$$

where $G(x, y, z)=\frac{-\left(x^{2}+y^{2}\right)}{\omega(z)^{2}}$ : is the complex electric field amplitude at the waist of the Gaussian beam, and

$$
\begin{aligned}
\varphi_{1}(x, y, z)=-(|\mathrm{I}| & +1) \arctan \frac{2 z}{k \omega_{0}{ }^{2}}+\frac{k\left(x^{2}+y^{2}\right)}{2 \mathrm{R}(z)}+1 \cdot \arctan \left(\frac{y}{x}\right) \\
& +k z
\end{aligned}
$$

\section{B. Multiplexing/De-multiplexing of OAM Beams}

OAM beams can be used to enable a variety of optical communication applications, such as multiplexing/de-multiplexing of information-carrying OAM beams to achieve an increase in capacity and spectral efficiency, and data exchange between OAM beams for flexible data processing [11]. An OAM beam has a helical (helicoidally) phase structure, when encoded with data information; the information-carrying OAM beam $\left(U_{S}(x, y, z, t)\right)$ can be described as:

$$
U_{S}(x, y, z, t)=S(t) * G(x, y, z) * \exp (i \varphi(x, y, z)
$$

where $S(t)$ is the applied data information.

For the multiplexing of $H$ information-carrying OAM-beam $\quad U_{S_{p}}(x, y, z, t)=S_{p}(t) * G_{p}(x, y, z) *$ $\exp \left(i \varphi_{p}(x, y, z)\right),(p=1,2,3, \ldots N)$, the resultant field is given by: 
$U_{M U X}(x, y, z, t)=\sum_{p=1}^{N} S_{p}(t) * G_{p}(x, y, z) * \exp \left(i \varphi_{p}(x, y, z)\right)(8)$

The phase $\varphi_{p}(x, y, z)$ expressed as;

$$
\begin{gathered}
\varphi_{p}(x, y, z)=-\left(\left|l_{p}\right|+1\right) \arctan \frac{2 z}{k \omega_{0}^{2}}+\frac{k\left(x^{2}+y^{2}\right)}{2 R(z)} \\
+l_{p} \cdot \arctan \left(\frac{y}{x}\right)+k z
\end{gathered}
$$

For the de-multiplexing of information-carrying OAM beams, we multiply the superposed OAM beams $U_{M U X}(\mathrm{x}, \mathrm{y}, \mathrm{z}, \mathrm{t})$ by $\exp \left(i \varphi_{q}(x, y, z)\right.$, with

$$
\begin{gathered}
\varphi_{q}(x, y, z)=-\left(\left|\left(-l_{q}\right)\right|+1\right) \arctan \frac{2 z}{k \omega_{0}^{2}}+\frac{k\left(x^{2}+y^{2}\right)}{2 R(z)} \\
+\left(-l_{q}\right) \cdot \arctan \left(\frac{y}{x}\right)+k z
\end{gathered}
$$

The result is given as:

$$
\begin{gathered}
U_{D E M U X}(x, y, z, t)=\exp \left(i \varphi_{q}(x, y, z)\right) * \sum_{p=1}^{N} S_{p}(t) * \\
G_{p}(x, y, z) * \exp \left(i \varphi_{p}(x, y, z)\right)
\end{gathered}
$$

\section{Mode Conversion}

To de-multiplex an OAM beam with a topological charge "l", an OAM decoder $\left(\exp \left(i \varphi_{q}(x, y, z)\right)\right.$ is used to convert back to a beam with a planar phase front. But when it was $\mathrm{N}$-OAM beams to carry, the phenomenon of interference between modes becomes very clear and the output of the decoder will not be a planar phase front. We can rewrite the equation $\mathrm{U}_{\text {DEMUX }}$, to better see this phenomenon, as follows:

$$
\begin{gathered}
U_{D E M U X}(x, y, z, t)=S_{q}(t) * G_{q}(x, y, z)+\sum_{p=1, p \neq q}^{N} S_{p}(t) * \\
G_{p}(x, y, z, t) * \exp \left(i \varphi_{p}^{\prime}(x, y, z)\right)(10)
\end{gathered}
$$

With

$$
\begin{gathered}
\varphi_{p}^{\prime}(x, y, z)=-\left(\left|\left(l_{p}-l_{q}\right)\right|+1\right) \arctan \frac{2 z}{k \omega_{0}^{2}}+\frac{k\left(x^{2}+y^{2}\right)}{2 R(z)} \\
+\left(l_{p}-l_{q}\right) \cdot \arctan \left(\frac{y}{x}\right)+k z
\end{gathered}
$$

We can see that only one of the superposed information carrying OAM beams (charge, $\ell_{q}$ ) is converted back to a beam with a planar phase front $\left(S_{q}(t) * G(x, y, z)\right)$, the others are still OAM beams, but with a topological charge $(l p-l q)$, so a new OAM beam will interfere with our desired beam.

\section{QPSK Demodulator}

For QPSK demodulator, a coherent demodulator is taken as an example (Fig. 4). In coherent detection technique the knowledge of the carrier frequency and phase must be known to the receiver. This can be achieved by using a PLL (phase lock loop) at the receiver. A PLL essentially locks to the incoming carrier frequency and tracks the variations in frequency and phase. For the following simulation, a PLL is not used but instead we simple use the output of the PLL.

For demonstration purposes we simply assume that the carrier phase recovery is done and simply use the generated reference frequencies at the receiver $(\cos (\omega t))$ and $(\sin (\omega t))$.

$$
\omega=2 \pi f_{c}, f_{c} \text { is the carrier frequency. }
$$

We consider in our theoretical simulation that the noises can be assimilated to an additive Gaussian noise (AWGN) with variance $\sigma_{b}^{2}$. In this case the received signal at the input of the OAM-DEMUX is the sum of $U_{M U X}(x, y, z, t)$ and noise (AWGN):

$$
r(t)=\sum_{p=1}^{N} S_{p}(t) * G_{p}(x, y, z) * \exp \left(i \varphi_{p}(x, y, z)\right)+b(t)
$$

After decodingr $(\mathrm{t})$ usingan OAM decoder, we obtain the following signal:

$$
r_{q}^{\prime}(t)=r(t) * \exp \left(i \varphi_{q}(x, y, z)\right)
$$

Hence:

$$
\begin{gathered}
r_{q}{ }^{\prime}(t)=S_{q}(t) * G_{q}(x, y, z)+\sum_{p=1, p \neq q}^{N} S_{p}(t) * G_{p}(x, y, z, t) * \\
\exp \left(i \varphi^{\prime}{ }_{p}(x, y, z)\right)+b(t) * \exp \left(i \varphi_{q}(x, y, z)\right)
\end{gathered}
$$

In the QPSK demodulator the received signal $r_{q}{ }^{\prime}(t)$ is multiplied by a reference frequency generators $(\cos (\omega t))$ and $(\sin (\omega t))$ on separate arms (in-phase and quadrature (IQ) arms).

The multiplied output on each arm is given by:

$$
\left\{\begin{array}{l}
I=r_{q}^{\prime}(t) * \cos (\omega t) \\
Q=r_{q}^{\prime}(t) * \sin (\omega t)
\end{array}\right.
$$

For a carrier frequency

$$
f_{c}=50 k H z\left\{\begin{array}{c}
I=r_{q}^{\prime}(t) \\
Q=r_{q}^{\prime}(t) * \sin (\omega t)
\end{array}\right.
$$

Finally the bits on the in-phase (I, even bits) arm and on the quadrature $(Q$, odd bits) arm are remapped to form detected information stream.

From the previous equations ((13) and (14)) we can extract two terms; one presents the interference (Int) and the second term shows the noise $(N)$ :

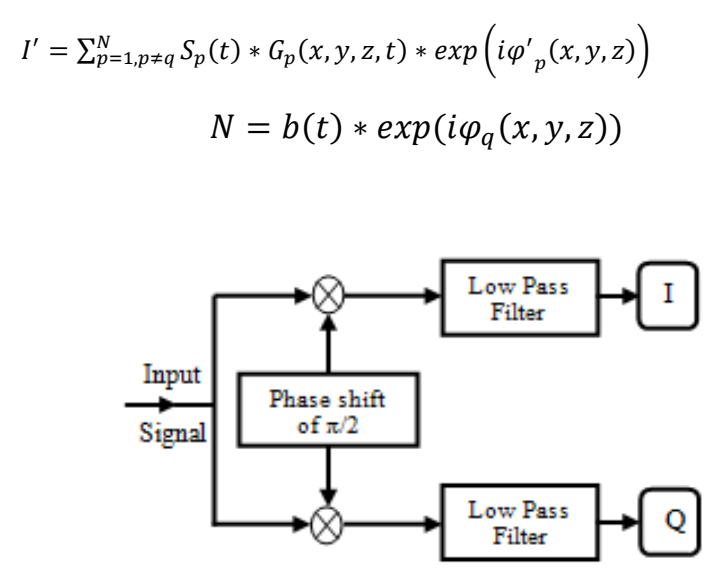

Fig. 4. QPSK Demodulator.

\section{TheOREtical Simulation of AN Optical}

\section{COMMUNICATION USING ORBITAL ANGULAR MOMENTUM}

This section presents a theoretical data transmission of OAM beams through an ideal optical fiber. Similar to wavelength-multiplexed networks, different users could occupy a different OAM states. A helix modulator block was designed to simulate the creation of helical phase front intertwining using an OAM encoder.

In Fig. 5 we present a block diagram of the theoretical 
setup for transmission of QPSK carrying 4 OAM beams. Four Gaussian beams $(\lambda=1550 \mathrm{~nm})$ with planar phase fronts, each carrying a QPSK signal are converted into four OAM $\left(\mathrm{OAM}_{+4}, \mathrm{OAM}_{+6}, \mathrm{OAM}_{+8}, \mathrm{OAM}_{+10}\right)$ with helical phase fronts using different OAM encoder.

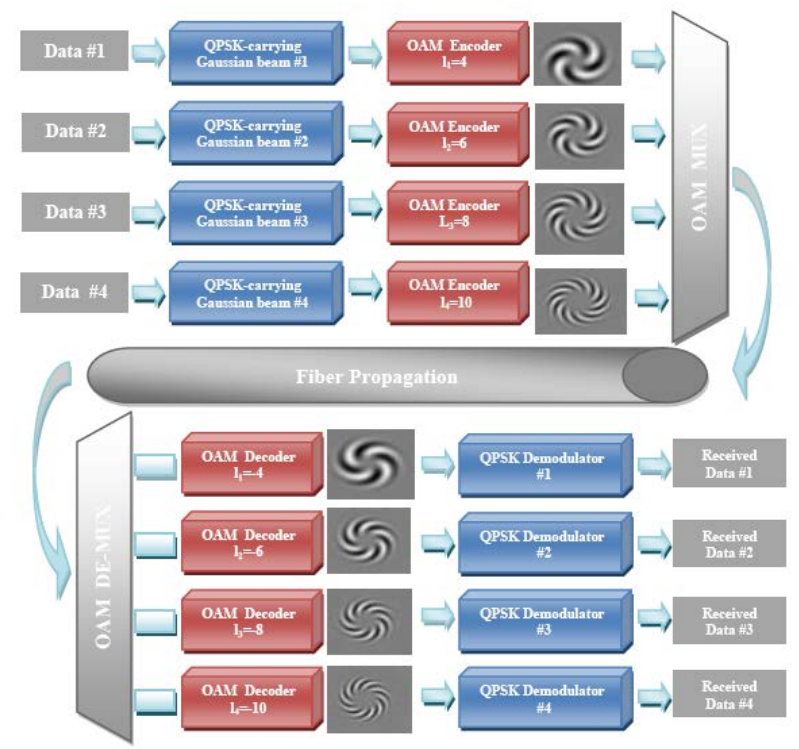

Fig. 5. Setup for transmission of QPSK carrying 4 OAM beams using an ideal channel.

After multiplexing, the OAM beams propagate in an ideal optical fiber. At the reception, a de-multiplexing of OAM beams used to de-multiplex one of the OAM beams back a beam with a planar phase front for coherent detection (Fig. 5).

We presented in Fig. 6 a 3D variation of the signal to interference ration $(S I R=S / I)$, and we can see that this ratio varies between 8 and $15 \mathrm{~dB}$. The performance of a wireless network critically depends on the signal to interference plus noise (SINR) levels at the receivers, presented by the following equation:

$$
S I N R=\frac{S}{N+I \prime}
$$

where $S$ is the desired signal power; $N$ is the noise power and $I$ ' the interference power. According to what is seen in Fig. 7 this ratio can be varied between 5 and $15 \mathrm{~dB}$.

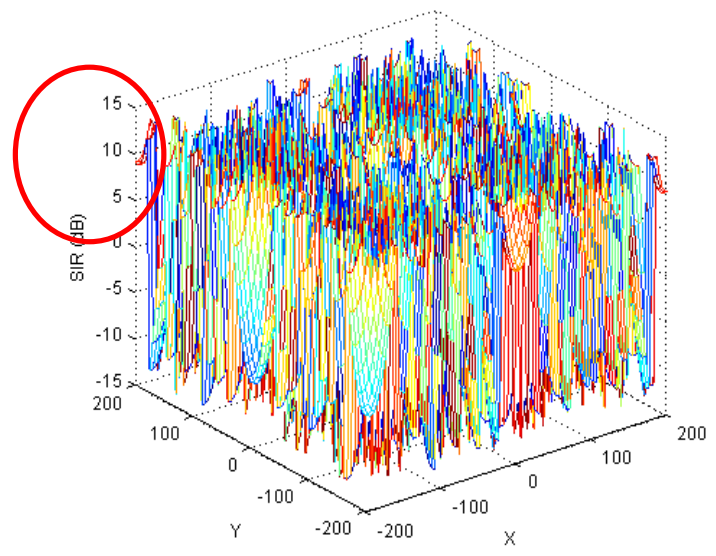

Fig. 6. 3Dpresentation of SIR=S/I.

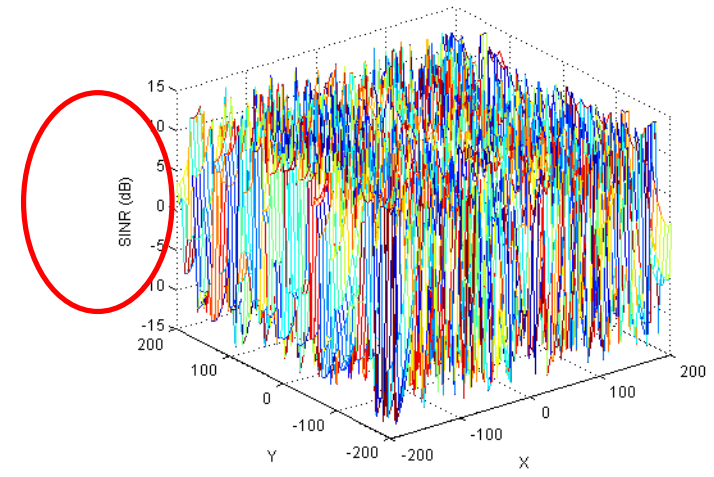

Fig. 7. 3Dpresentation of $S I N R=S /(I+N)$.

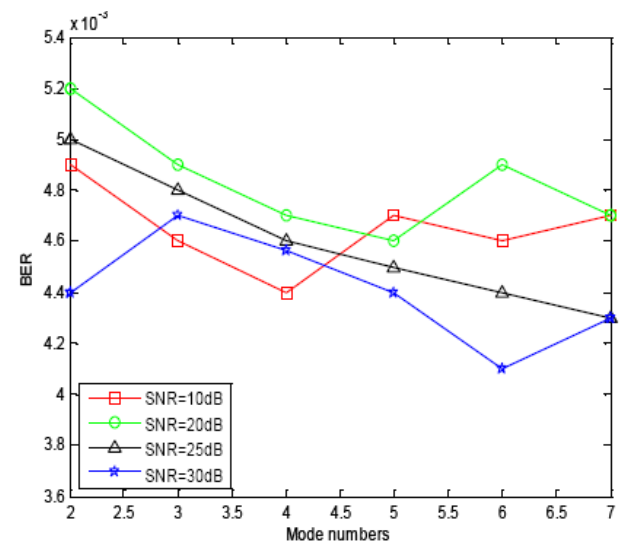

Fig. 8. BER versus mode numbers for different $S N R$.

The simulation presented in this paper has been carried out in MATLAB to evaluate the performance of propagation with different OAM state. Bit Error Rate plots in our system to examine the errors introduced by the different blocks of the data transmission chain; the OAM encoder and multiplexing/de-multiplexing block. BER were measured for different SNR value as a function of mode number.

The evolution of the BER performance of our system presented in Fig. 8, using the different equations developed in section II and III, with the same parameters. This performance was evaluated as a function of the number of OAM modes, at a wavelength $\lambda$ equal to $1550 \mathrm{~nm}$ for different SNR. From this figure we can observe that the performance is good whatever the number of mode, it is always less than 5.2 $\times 10^{-3}$, and the best performance was obtained when SNR $=30 \mathrm{~dB}$. We can see also that the number of modes has not a great effect on the performance of the system.

\section{CONCLUSION}

In this paper we proposed a theoretical simulation of a QPSK carrying OAM beams through the different blocks of the transmission chain derived here (OAM encoder, multiplexing and de-multiplexing). We also evaluate the performance of our system and we have seen that the BER $<5.7 \times 10^{-3}$. In future work, we will try to simulate include optical channel effect whether free space or optical fiber based.

\section{ACKNOWLEDGEMENTS}

This research is funded and supported by the KACST-TIC 
in Radio Frequency and Photonics (RFTONICS). King Saud University, Saudi Arabia, in addition to "SysCom" laboratory, the National School of Engineer Tunis, Tunisia.

\section{REFERENCES}

[1] J. Wang et al., "Demonstration of 12.8-bit/s/Hz spectral efficiency using 16-QAM signals over multiple orbital-angular momentum modes," in Proc. the European Conference on Optical Communications, Geneva (Optical Society of America), 2011.

[2] J. Wang et al.,“25.6-bit/s/Hz spectral efficiency using 16-QAM signals over pol-muxed multiple orbital-angular-momentum modes," in Proc. the IEEE Photonics Conference, Denver (IEEE Photonics Society), 2011.

[3] I. M. Fazal et al., "Demonstration of 2-Tbit/s data link using orthogonal orbital-angular-momentum modes and WDM," in Proc. the Frontiers in Optics, San Jose (Optical Society of America), 2011.

[4] J. Wang et al.,"Experimental demonstration of 100-Gbit/s DQPSK data exchange between orbital-angular-momentum modes," in Proc. of the Optical Fiber Communication Conference, Los Angeles (Optical Society of America), 2012.

[5] A. H. Gnauck et al.,"Spectrally efficient long-haul WDM transmission using 224-Gb/s polarization-multiplexed 16-QAM,”J. Lightwave Technol., vol. 29, pp. 373-377, 2011.

[6] A. Sano et al., "Ultra-high capacity WDM transmission using spectrally-efficient PDM 16-QAM modulation and C- and extended L-band wideband optical amplification,” J. Lightwave Technol., vol. 29, pp. 578-586, 2011.

[7] T. Richter et al.,"Transmission of single-channel 16-QAM data signals at terabaud symbol rates," J. Lightwave Technol., vol. 30, pp. 504-511, 2012.

[8] X. Liu et al., "1.12-Tb/s 32-QAM-OFDM super channel with 8.6-b/s/Hz intrachannel spectral efficiency and space-division multiplexed transmission with $60-\mathrm{b} / \mathrm{s} / \mathrm{Hz}$ aggregate spectral efficiency," Opt. Express 19, B958-B964, 2011.

[9] R. Ryf et al., "Mode-division multiplexing over $96 \mathrm{~km}$ of few-mode fiber using coherent 6 × 6 MIMO processing," J. Lightwave Technol., vol. 30, pp. 521-531, 2012.

[10] G. Gibson, et al., "Freespace information transfer using light beams carrying orbital angular momentum,” Opt. Express 12, pp. 5448-5456, 2004.

[11] J. Wang et al.,"Terabit free-space data transmission employing orbital angular momentum multiplexing," Nature Photonics, vol. 6, 2012

[12] Y. Yue et al., "1.6-Tbit/s Muxing, Transmission and Demuxing through 1.1- km of vortex fiber carrying 2 OAM beams each with 10 wavelength channels," Optical Society of America, 2013.

[13] N. Bozinovic et al., "Terabit-scale orbital angular momentum mode division multiplexing in fibers," American Association for the Advancement of Science, vol. 340, pp. 1545-1548, 2013.

[14] P. Boffi, P. Martelli, A. Gatto, and M. Martinelli, "Mode-division multiplexing in fibre-optic communications based on orbital angular momentum," Journal of Optics, 2013.
[15] X. H. Lu, H. Q. Huang, C. L. Zhao, J. F. Wang, and H. Chen, “Optical vortex beams and optical votices," Laser \& Optoeletronics Progress, pp. 50-56, 2008.

[16] Y. Y. Zhu, L.Wei, J. C. Zhang, M. J. Jing, J. Li, and X. W. Guan, "Orbital angular momentum density distribution and its simulation analysis of gauss vortex beam," in Proc. International Conference on Optical Instruments and Technology: Optical Trapping and Microscopic Imaging, 2009.

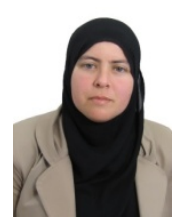

Besma Hammami was born in Bizerte, Tunisia, in 1984. She received the B.E. degree in telecommunication engineering, M.E. degree in communication system from the National Engineering School of Tunis in 2008 and 2010 respectively. She is currently a Ph.D. student in telecommunication, affiliated to the SYSCOM Laboratory at National School of Engineering of Tunisia (ENIT). Her research activity is in optical communications.

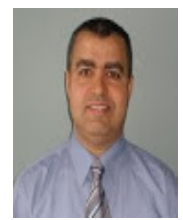

Habib A. Fathallah received the B.S.E.E. degree from the National Engineering School of Tunis in1994 and the M.S.C. and Ph.D. degrees in electrical engineering from Université Laval, Québec, Canada, in 1997 and 2001, respectively. He initiated the use of Bragg Gratings technology for all-optical/all-fiber coding/decoding in optical CDMA systems. He was the founder of Access Photonic Networks (2001-2006), an adjunct professor with the Electrical and Computer Engineering Department of Laval University (Quebec, Canada) (2003-2012). He is currently with the Electrical Engineering Department and the KACST-Technology Innovation Center in Radio Frequency and Photonics for the e-Society, College of Engineering, King Saud University (Riyadh, Saudi Arabia) and his research interests include photonics and optical communications systems, optical sensing, OCDMA, PONs, FiWi, complex modulation, optical space and mode division multiplexing, orbital angular momentum multiplexing, electromagnetic energy harvesting, and wireless powered systems.

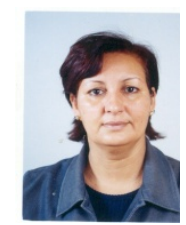

Houria Rezig was born in Casablanca, Morocco, in 1955. She received the Ph.D. degrees in electrical engineering from University of Science and Techniques of Montpellier (Electronic-Optoelectronic Center of Montpellier) France, in 1984. She is currently a full professor in 'telecommunications", at National School of Engineering of Tunisia (ENIT). She's affiliated to SYSCOM Laboratory at ENIT and head of a research group 'optical communications', since 2005. She's responsible of numbers of Research Projects in the frame of Bilateral Tunisian French Scientific Cooperation. She has been a member IEEE since 10 years. Her basic lectures and research activities interests in electronics, optoelectronics, integrated optics and optical communication systems. 